\title{
Sequence MAP Decoding of Trellis Codes for Gaussian and Rayleigh Channels
}

\author{
Saud A. Al-Semari, Fady Alajaji, Member, IEEE, and Tom Fuja, Senior Member, IEEE
}

\begin{abstract}
This paper considers the use of sequence maximum a posteriori (MAP) decoding of trellis codes. A MAP receiver can exploit any "residual redundancy" that may exist in the channelencoded signal in the form of memory and/or a nonuniform distribution, thereby providing enhanced performance over very noisy channels, relative to maximum likelihood (ML) decoding.

The paper begins with a first-order two-state Markov model for the channel encoder input. A variety of different systems with different source parameters, different modulation schemes, and different encoder complexities are simulated. Sequence MAP decoding is shown to substantially improve performance under very noisy channel conditions for systems with low-to-moderate redundancy, with relative gain increasing as the rate increases. As a result, coding schemes with multidimensional constellations are shown to have higher MAP gains than comparable schemes with two-dimensional (2-D) constellations.

The second part of the paper considers trellis encoding of the code-excited linear predictive (CELP) speech coder's line spectral parameters (LSP's) with four-dimensional (4-D) QPSK modulation. Two source LSP models are used. One assumes only intraframe correlation of LSP's while the second one models both intraframe and interframe correlation. MAP decoding gains (over ML decoding) as much as $4 \mathrm{~dB}$ are achieved. Also, a comparison between the conventionally designed codes and an I-Q QPSK scheme shows that the I-Q scheme achieves better performance even though the first (simpler) LSP model is used.
\end{abstract}

Index Terms - AWGN and Rayleigh channels, CELP speech coding, Markov sources, sequence MAP decoding, source redundancy, trellis codes.

\section{INTRODUCTION}

$\mathbf{T}$ RADITIONALLY, source coding (data compression) and channel coding (error control) are designed independently of one another. This is justified by Shannon's separation principle [1], which states that no performance degradation is suffered if the two functions are thus partitioned. However, this principle is an asymptotic result permitting unlimited delay and complexity. Given a limited decoding complexity/delay, joint source-channel coding could outperform separately designed pairs.

Manuscript received June 10, 1997; revised February 24, 1998. This work was supported in part by King Fahd University of Petroleum and Minerals, Saudi Arabia, the Natural Sciences and Engineering Research Council of Canada (NSERC) under Grant OGP0183645, and the NSF and U.S. Department of Defense.

S. A. Al-Semari is with the Electrical Engineering Department, King Fahd University of Petroleum and Minerals, Dhahran 31261, Saudi Arabia.

F. Alajaji is with the Department of Mathematics and Statistics and the Department of Electrical and Computer Engineering, Queen's University, Kingston, Ont., K7L 3N6, Canada.

T. Fuja is with the Electrical Engineering Department, University of Notre Dame, Notre Dame, IN 46556 USA.

Publisher Item Identifier S 0018-9545(99)04069-4.
Some recent work in this area has investigated the design of source codes that are robust to channel errors [2]-[4]. Conversely, the work in [5]-[7] has concentrated on the design of channel decoders that exploit the known characteristics of the source code. The work here focuses on the performance of trellis codes with sequence maximum a posteriori (MAP) decoding of correlated signals transmitted over very noisy additive white Gaussian noise (AWGN) and Rayleigh channels.

An ideal source encoder would compress an audio, image, or a data signal to produce an independent, identically distributed (i.i.d.) sequence of bits at a rate equal to the entropy rate of the source. Most source coding algorithms are not ideal; the output bit stream contains residual redundancy in the form of memory and/or nonuniform distribution, and this redundancy is thus present at the input of the channel encoder.

This residual redundancy may be exploited at the receiver by adjusting the Viterbi algorithm decoding metric to use the source's a priori probabilities. Sequence MAP Viterbi decoders take advantage of this residual redundancy to enhance the performance of the system under very noisy channel conditions. This decoding method used in conjunction with other means (i.e., soft-decision decoding and channel state information estimation) results in a very robust system under bad channel conditions.

The traditional Viterbi algorithm selects the maximum likelihood (ML) sequence as its estimate; it does not minimize the probability of error of the data bits [8]-[10]. However, its performance is very close to that of the optimal symbolby-symbol decoding algorithm [8]. Furthermore, although the existing symbol-by-symbol decoding algorithms apply only to linear codes, the Viterbi algorithm is applicable to linear and nonlinear codes. Sequence MAP decoding does not require substantial modification to the Viterbi algorithm for trelliscoded systems, and, as indicated in [5] and [6], may be used if needed where a bad channel environment exists. In [5], Hagenauer showed that $2-3-\mathrm{dB}$ gains could be obtained for pulse code modulation (PCM) transmission and the full rate global system for mobile (GSM) speech codec. A 16-state rate $1 / 2$ convolutional code with BPSK modulation and a dynamic two-state Markov correlation estimator were used. In [6], Alajaji et al. used both block and convolutional codes to exploit the residual redundancy in the CELP speech encoder. A 32-state rate 3/4 convolutional code with BPSK modulation and sequence MAP decoding were implemented. The receiver was supplied with the source a priori information which was obtained using first and second-order Markov models on a 
large training sequence from the TIMIT [11] speech database. Decoding gains of $2-5 \mathrm{~dB}$ were achieved.

The work in this paper is divided into two main parts. The first part assumes a simple ideal first-order two-state Markov source model. This simple model is chosen because its parameters may be easily estimated at the receiver. A variety of different systems with different sources, modulation schemes, and trellis code complexities are simulated. Extensive simulations of these system configurations are performed to assess their effect on the sequence MAP decoding gains ${ }^{1}$ (simply called MAP gains) over ML decoding. In performing such simulations, we try to address the following questions.

- What is the effect of increasing the trellis encoder memory?

- What is the effect of increasing the encoder rate?

- What is the effect of increasing the signal constellation?

- What is the effect of the redundancy type?

- What is the difference in MAP decoding gains for coded as well as uncoded systems?

- What is the effect of the channel type-AWGN or Rayleigh?

The first part is concluded with an example comparing a two-dimensional (2-D) and a four-dimensional (4-D) QPSK TCM schemes with the same spectral efficiencies and ML asymptotic coding gains. It is shown that increasing the dimensionality (i.e., multiplicity) of the code results in increased MAP (versus ML) decoding gains.

In the second part, we extend the results of [12] by considering encoding the line spectral parameters (LSP's) of FS-1016 CELP using trellis codes with 4-D QPSK modulation. Both AWGN and Rayleigh channels are considered.

\section{SEQuence MAP DeCoding for IdeAl Sources}

\section{A. System Model}

The basic model block diagram is shown in Fig. 1. The information bit sequence $\left\{u_{1}, u_{2}, \ldots\right\}$ is modeled by a stationary first-order two-state Markov process as shown in Fig. 2. The sequence $\left\{u_{1}, u_{2}, \ldots\right\}$ represents the output of a source encoder, or (if the source is not compressed) the output of the source itself. We denote the transition probabilities $\operatorname{Pr}\left(u_{i}=0 \mid u_{i-1}=0\right)$ and $\operatorname{Pr}\left(u_{i}=1 \mid u_{i-1}=1\right)$ by $\operatorname{Pr}(0 \mid 0)$ and $\operatorname{Pr}(1 \mid 1)$, respectively.

The entropy of the Markov chain can be computed to arrive at an estimate of the source redundancy. Let $H_{\infty}(U)$ denote the source entropy rate and let $H(U)$ denote the entropy of a memoryless source with the same marginal distribution as the source. Define [12]

$$
\begin{aligned}
\rho_{D} & \triangleq 1-H(U) \\
\rho_{M} & \triangleq H(U)-H_{\infty}(U) \\
\rho_{T} & \triangleq \rho_{D}+\rho_{M}=1-H_{\infty}(U) .
\end{aligned}
$$

Then $\rho_{D}$ denotes the redundancy due to the nonuniform distribution of the source, $\rho_{M}$ denotes the redundancy due

\footnotetext{
${ }^{1}$ We use the term "decoding gains" instead of "coding gains" since we are comparing two systems with the same codes but different decoders.
}

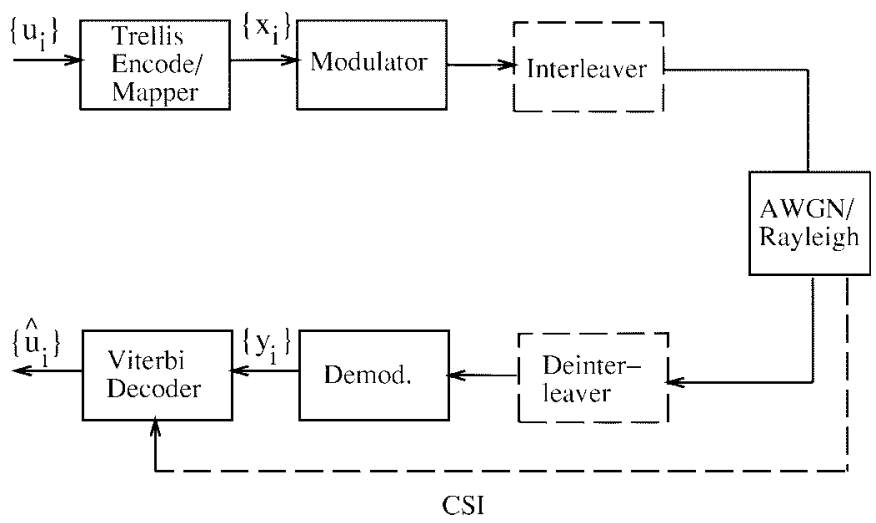

Fig. 1. Block diagram of the system model.

to the source memory, and $\rho_{T}$ is the total source redundancy. The two forms of redundancy are utilized by the decoder to combat channel errors.

The source bits are arranged in a sequence of binary $k$-tuples $\left\{\underline{u}_{1}, \underline{u}_{2}, \ldots\right\}$. At time $i, \underline{u}_{i}$ is an input to a trellis encoder which produces a $(k+1)$-tuple of binary bits $\underline{c}_{i}$. The trellis encoder output $\underline{c}_{i}$ is mapped to $\underline{x}_{i}=\left\{x_{i}^{1}, \ldots, x_{i}^{m}\right\}$, a vector of $m$ complex numbers, where $m$ is the number of transmitted signals per trellis branch-i.e., the multiplicity of the code. The sequence $\mathrm{x}_{N}=\left\{\underline{x}_{1}, \ldots \underline{x}_{N}\right\}$ is transmitted over the channel. This is described by

$$
y_{i}^{j}=a_{i}^{j} x_{i}^{j}+n_{i}^{j}, \quad \text { with } 1 \leq i \leq N, \quad \text { and } \quad 1 \leq j \leq m
$$

where $n_{i}^{j}$ is a 2-D zero-mean additive Gaussian noise with a single-sided power spectral density of $N_{o}$. The variable $a_{i}^{j}$ depends on the channel assumption.

- For a purely AWGN nonfading channel, $a_{i}^{j}=1$.

- For a fully interleaved Rayleigh fading channel, we assume $\left\{a_{i}^{j}\right\}$ is a sequence of i.i.d. Rayleigh distributed random variables with $E\left[\left(a_{i}^{j}\right)^{2}\right]=1$. Note that the assumption of full interleaving is justified since under very noisy channel conditions (the region of interest in this work) interleaving requirements are much less than at high signal-to-noise ratios (SNR's). At low SNR, the additive noise dominates any effects due to possible correlation in the fading.

\section{B. MAP Decoding Rule}

The sequence MAP decision rule [12] is to choose $\hat{\mathbf{x}}_{N}$ which maximizes

$$
f\left(\mathbf{y}_{N} \mid \hat{\mathbf{x}}_{N}\right) \operatorname{Pr}\left(\hat{\mathbf{x}}_{N}\right) .
$$

For the AWGN channel, this reduces to choosing $\hat{\mathbf{x}}_{N}$ which minimizes

$$
\sum_{i=1}^{N} \sum_{j=1}^{m}\left|y_{i}^{j}-\hat{x}_{i}^{j}\right|^{2}-N_{o} \ln \operatorname{Pr}\left(\hat{\mathbf{x}}_{N}\right) .
$$

Using the chain rule and first-order Markov source property, $\ln \operatorname{Pr}\left(\hat{\mathbf{x}}_{N}\right)$ can be expressed as

$$
\ln \operatorname{Pr}\left(\hat{\mathbf{x}}_{N}\right)=\ln \operatorname{Pr}\left(\hat{\mathbf{u}}_{N}\right)
$$




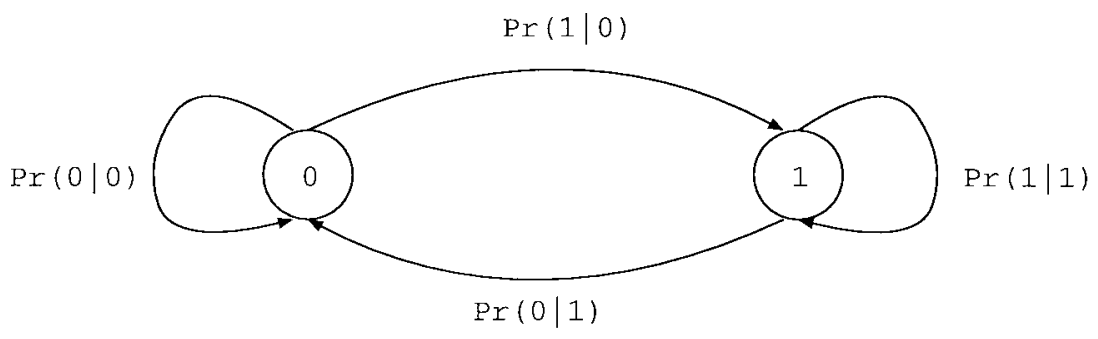

Fig. 2. Two-state binary Markov source model.

$$
\begin{aligned}
& =\sum_{i=1}^{N} \ln \operatorname{Pr}\left(\underline{\hat{u}}_{i} \mid \underline{\hat{u}}_{i-1}, \underline{\hat{u}}_{i-2}, \ldots, \underline{\hat{u}}_{1}\right) \\
& =\sum_{i=1}^{N} \ln \operatorname{Pr}\left(\underline{\hat{u}}_{i} \mid \underline{\hat{u}}_{i-1}\right) .
\end{aligned}
$$

Therefore, the MAP decoding rule is to choose $\hat{\mathbf{x}}_{N}$ which minimizes

$$
\sum_{i=1}^{N}\left(\sum_{j=1}^{m}\left|y_{i}^{j}-\hat{x}_{i}^{j}\right|^{2}-N_{o} \ln \operatorname{Pr}\left(\underline{\hat{u}}_{i} \mid \underline{\hat{u}}_{i-1}\right)\right) .
$$

For the Rayleigh distributed fading channel, the fading values-i.e., the channel state information (CSI) - is incorporated into the decoding metric to choose $\hat{\mathbf{x}}_{N}$ minimizing

$$
\sum_{i=1}^{N}\left(\sum_{j=1}^{m}\left|y_{i}^{j}-a_{i}^{j} \hat{x}_{i}^{j}\right|^{2}-N_{o} \ln \operatorname{Pr}\left(\underline{\hat{u}}_{i} \mid \underline{\hat{u}}_{i-1}\right)\right) \text {. }
$$

\section{Bit Error Probability Upper Bound}

Using the union bound approach, the average bit error probability $P_{b}$ of trellis-coded systems may be upperbounded as [13]

$$
P_{b} \leq \sum_{n=1}^{\infty} \sum_{\mathbf{x}_{n}} \sum_{\mathbf{x}_{n} \neq \hat{\mathbf{x}}_{n}} N_{n}\left(\mathbf{x}_{n}, \hat{\mathbf{x}}_{n}\right) \operatorname{Pr}\left(\mathbf{x}_{n}\right) P\left(\mathbf{x}_{n} \rightarrow \hat{\mathbf{x}}_{n}\right)
$$

where $P\left(\mathbf{x}_{n} \rightarrow \hat{\mathbf{x}}_{n}\right)$ is the pairwise error probability between the sequences $\mathbf{x}_{n}$ and $\hat{\mathbf{x}}_{n}, \operatorname{Pr}\left(\mathbf{x}_{n}\right)$ is the a priori probability of transmitting the sequence $\mathbf{x}_{n}$, and $N_{n}\left(\mathbf{x}_{n}, \hat{\mathbf{x}}_{n}\right)$ is the number of bit errors when the transmitted sequence $\mathbf{x}_{n}$ is decoded as $\hat{\mathbf{x}}_{n}$. For the AWGN channel, $P\left(\mathbf{x}_{n} \rightarrow \hat{\mathbf{x}}_{n}\right)$ can be expressed as [7]

$$
P\left(\mathbf{x}_{n} \rightarrow \hat{\mathbf{x}}_{n}\right)=Q\left(\sqrt{\frac{d_{E}^{2}}{2 N_{o}}}-\frac{N_{o}}{2} \ln \left(P\left(\hat{\mathbf{x}}_{n}\right) / P\left(\mathbf{x}_{n}\right)\right) / d_{E}\right)
$$

where $d_{E}^{2}$ is the squared Euclidean distance between $\mathbf{x}_{n}$ and $\hat{\mathbf{x}}_{n}$. In enumerating the trellis paths, the super-state diagram with $2^{2 \nu}$ states is used, where $\nu$ is the number of memory elements of the minimal encoder [13]. It is not possible to use the reduced state diagram of the code for path enumeration even if the code satisfies the uniformity properties. This is because not all sequences are equiprobable, and, hence, the paths will contribute unequally to the upper bound.
TABLE I

The Four Source Models and Their Redundancies

\begin{tabular}{c|c|c|c|c|c}
\hline Source & $\operatorname{Pr}(0: 0)$ & $\operatorname{Pr}(1: 1)$ & $\rho_{I}$ & $\rho_{M}$ & $\rho_{T}$ \\
\hline I & 0.8 & 0.2 & 0.278 & 0.0 & 0.278 \\
\hline II & 0.8 & 0.8 & 0.0 & 0.278 & 0.278 \\
\hline III & 0.9 & 0.8 & 0.082 & 0.365 & 0.447 \\
\hline IV & 0.2 & 0.9 & 0.496 & 0.007 & 0.503 \\
\hline
\end{tabular}

However, the union bound with either ML [14] or MAP [5] decoding is loose at high decoding error rates $\left(\sim 10^{-2}\right)$. It does not provide useful quantitative values for the MAP decoding gains. Some efforts on the error probability analysis of trellis codes at low SNR have appeared. For the BSC, a Markovian technique was used to exactly determine the probability of error of trellis codes with hard decision ML decoding [15]. However, the method is computationally expensive even for two-state codes. Another approach appeared in [16], where modifications of the union bound were described and very tight upper and lower bounds for the error probability with ML decoding were given. However, computational complexity again limits its use.

Another improved upper bound on the error event probability $P_{e}$ for the Viterbi decoder over the binary symmetric channel (BSC) was presented in [17]. At high error rates, this upper bound does not give trivial values for $P_{e}$, as in the case of the standard union bound. Also, it may be deduced from [17] that a higher length of an error event path contributes to the reduction of $P_{e}$. In this work, we resort to computer simulations to accurately assess the performance of the MAP decoder.

\section{Simulated SySTEMS}

A wide variety of system configurations have been simulated. The source is assumed to have one of four distributions. Table I shows these distributions with their respective redundancies. The first source is a nonuniformly distributed i.i.d. process. The second is a symmetric binary Markov source. These two sources have the same total redundancy but in completely different forms; the i.i.d. source exhibits all its redundancy in its nonuniform distribution, while the symmetric Markov source exhibits all its redundancy in the form of memory. The last two distributions are asymmetric Markov sources; they are highly skewed to one form of redundancy.

BPSK, QPSK, and 8-PSK modulation schemes were used; in each case, coherent detection is assumed. Trellis codes with different encoder rates and complexities were imple- 
mented. These different configurations are tested on two channel models - a pure nonfading AWGN channel and a Rayleigh-distributed fading channel model with ideal interleaving. These two channel models describe the extreme cases of channels encountered in practice. Hence, the results obtained over other channels (e.g., Rician) will lie in between our results.

For comparison purposes, uncoded BPSK-modulated systems were simulated. Four different sources were used over both the Gaussian and Rayleigh channels. A two-state Viterbi decoder with sequence MAP detection was used. MAP detection gains, compared to symbol-by-symbol ML detection, were highly affected by the type of redundancy. Uncoded systems with redundancy in the form of memory have higher MAP decoding gains than those with all or most of their redundancy due to a nonuniform distribution.

In coded systems, the chosen codes are optimized for the channel model assumed. The code design criterion for the AWGN channel is to maximize the minimum Euclidean distance of the code. For the Rayleigh channel, the design criterion is to maximize the minimum time diversity of the code-i.e., its minimum Hamming distance in signal symbols.

\section{A. BPSK-Modulated Systems}

For systems with BPSK modulation, two families of trellis codes were used-rate $1 / 2$ and rate $2 / 3$ codes. Both families of codes were obtained from [18] to maximize Hamming distance; for trellis codes with BPSK modulation, there is a one-to-one correspondence between the code's minimum Euclidean distance $d_{\text {free }}$ and its minimum Hamming distance $d_{H}$-i.e, $d_{\text {free }}=4 E_{s} d_{H}$. Therefore, the codes are optimized for both channels.

Rate 1/2 codes with 4-, 8-, and 16-state encoders were used for the four source models. Their minimum Hamming distances are five, six, and seven, respectively. Their minimum normalized Euclidean distances (i.e., $d_{\text {free }} / E_{s}$ ) are accordingly 20,24 , and 28, respectively. The bandwidth efficiency of these codes are $0.5 \mathrm{~b} / \mathrm{s} / \mathrm{Hz}$. A decoding buffer length of $8 \nu$ was used, where $\nu$ is the number of memory elements in the encoder. At a decoding bit error rate (BER) of 0.02, MAP decoding gains (over ML decoding) are as high as $1.4 \mathrm{~dB}$ for the Gaussian channel and $2 \mathrm{~dB}$ for the Rayleigh channel for a correlated source with about $50 \%$ source redundancy.

Rate 2/3 codes with 4-, 8-, and 16-state encoders were also used to show the effect of increasing the encoder rates; higher MAP decoding gains were obtained. For example, at a decoding BER of 0.02 , gains (MAP versus ML) are as high as $2.5 \mathrm{~dB}$ for the Gaussian channel and $4.0 \mathrm{~dB}$ for the Rayleigh channel for a correlated source with about $50 \%$ source redundancy.

\section{B. QPSK-Modulated Systems}

For QPSK-modulated systems, the same rate $1 / 2$ trellis codes described above were used. However, each two-tuple of bits is mapped to 2-D QPSK signal points. Gray mapping was used so that maximizing the minimum Hamming distance corresponds to maximizing the minimum Euclidean distance.
Moreover, using this approach the codes are optimized for both channels. The spectral efficiency in this case is $1 \mathrm{~b} / \mathrm{s} / \mathrm{Hz}$. Encoders with 4, 8, and 16 states were used. Their minimum Hamming distances (in symbols) are 3, 4, and 5, respectively, and the normalized minimum Euclidean distances are 10, 12, and 14, respectively. A decoding buffer length of $8 \nu$ was used for these codes. The gains are very close to that of rate $1 / 2$ codes with BPSK modulation. The reason for this similarity is because the two systems have the same encoder rates and QPSK signals have the same error performance as BPSK signals.

\section{8-PSK-Modulated Systems}

Octal PSK modulation was also simulated to see the effect of increasing the signal constellation. This system was constructed using rate 2/3 codes with natural 8-PSK mapping; Ungerboeck's 8- and 16-state codes were used [19]. These code are (fortunately) optimum for both the Gaussian and the Rayleigh channels [20]. The minimum Hamming distances (in symbols) are two and three, respectively, and the minimum Euclidean distances are 4.586 and 5.172, respectively [20]. A decoding buffer length of $8 \nu$ was used for these codes. Higher MAP decoding gains than that of rate $2 / 3$ codes with BPSK are obtained. For example, at a decoding BER of 0.02 gains (MAP versus ML) are as high as $3.3 \mathrm{~dB}$ for the Gaussian channel and $4.6 \mathrm{~dB}$ for the Rayleigh channel for a correlated source with about $50 \%$ source redundancy.

\section{Observations and Discussions}

To determine the performance of the different codes, extensive simulations were performed. Tables II-V show the MAP decoding gains (versus ML decoding) for all different systems at BER's of $0.1,0.05,0.02$, and 0.01 . We first notice that the gains diminish as the BER decreases. The diminishing rate is slower for uncoded systems. At lower error rates, ML decoding will have only a very slight degradation in error performance compared to sequence MAP decoding. This suggests that, to reduce the decoding computations, MAP decoding should only be used when the channel is sufficiently bad.

We notice also that the gains for the Rayleigh channel are significantly higher than the corresponding gains for the AWGN channel. This is due to the relatively gradual slope of the BER curves for the Rayleigh system. This is very clear for uncoded systems where coding substantially improves the performance over Rayleigh channels.

Comparisons for the uncoded systems reveal that the type of redundancy significantly affects the decoding gains, especially for the Rayleigh channel. Redundancy in the form of memory results in larger MAP decoding gains than does redundancy in the form of nonuniform distribution. Another remark is that the gains does not diminish quickly as the BER decreases. This suggests that even at low error rates $\left(\sim 10^{-3}\right)$ appreciable gains over the Rayleigh channels may be obtained.

For the coded systems, it can be seen that the rate $1 / 2$ codes with BPSK and QPSK modulations give comparable gains. This is due to the fact that the same encoder is used and Graymapped QPSK has the same error performance as BPSK. It can 
TABLE II

MAP DeCoding Gains for Different TRellis Codes over AWGN (Rayleigh) Channels. Source I [with Distribution $\operatorname{Pr}(0 \mid 0)=.8, \operatorname{Pr}(1 \mid 1)=.2$ ]

\begin{tabular}{c|c|c|c||c|c|c|c}
\hline $\begin{array}{c}\text { Modulation } \\
\text { Type }\end{array}$ & \multirow{2}{*}{ Rate } & \multicolumn{1}{|c||}{$\begin{array}{c}\text { Spectral } \\
\text { Efficiency }\end{array}$} & \multicolumn{1}{l||}{} & \multicolumn{4}{|c}{ Gains (BER) } \\
\cline { 5 - 8 } & & $10 \%$ & $5 \%$ & $2 \%$ & $1 \%$ \\
\hline BPSK & uncoded & 1 & - & $0.9(2.1)$ & $0.5(1.6)$ & $0.4(1.3)$ & $0.3(1.2)$ \\
\hline \hline BPSK & $1 / 2$ & $1 / 2$ & 2 & $1.2(1.6)$ & $0.9(1.1)$ & $0.6(0.8)$ & $0.5(0.7)$ \\
\hline BPSK & $1 / 2$ & $1 / 2$ & 3 & $1.2(1.7)$ & $0.9(1.2)$ & $0.7(0.9)$ & $0.5(0.8)$ \\
\hline BPSK & $1 / 2$ & $1 / 2$ & 4 & $1.4(1.8)$ & $1.0(1.4)$ & $0.8(1.1)$ & $0.5(0.9)$ \\
\hline BPSK & $2 / 3$ & $2 / 3$ & 2 & $2.4(3.5)$ & $1.7(2.6)$ & $1.1(1.9)$ & $.8(1.6)$ \\
BPSK & $2 / 3$ & $2 / 3$ & 3 & $2.5(3.6)$ & $1.7(2.6)$ & $1.1(1.9)$ & $1.0(1.6)$ \\
\hline BPSK & $2 / 3$ & $2 / 3$ & 4 & $2.4(3.6)$ & $1.7(2.7)$ & $1.3(2.2)$ & $1.0(1.9)$ \\
\hline \hline QPSK & $1 / 2$ & 1 & 2 & $1.2(1.5)$ & $0.8(1.1)$ & $0.6(0.8)$ & $0.5(0.6)$ \\
\hline QPSK & $1 / 2$ & 1 & 3 & $1.2(1.5)$ & $0.8(1.1)$ & $0.7(0.9)$ & $0.5(0.7)$ \\
\hline QPSK & $1 / 2$ & 1 & 4 & $1.3(1.8)$ & $1.0(1.4)$ & $0.8(1.1)$ & $0.5(1.0)$ \\
\hline 8PSK & $2 / 3$ & 2 & 3 & $3.2(3.8)$ & $2.4(2.8)$ & $1.7(2.1)$ & $1.4(1.9)$ \\
\hline 8PSK & $2 / 3$ & 2 & 4 & $3.2(3.9)$ & $2.4(3.0)$ & $1.9(2.5)$ & $1.5(2.1)$ \\
\hline
\end{tabular}

TABLE III

MAP DeCoding Gains for Different Trellis Codes over AWGN (Rayleigh) Channels. Source II [with Distribution $\operatorname{Pr}(0 \mid 0)=.8, \operatorname{Pr}(1 \mid 1)=.8$ ]

\begin{tabular}{|c|c|c|c|c|c|c|c|}
\hline \multirow{2}{*}{$\begin{array}{c}\text { Modulation } \\
\text { Type }\end{array}$} & \multirow[t]{2}{*}{ Rate } & \multirow{2}{*}{$\begin{array}{l}\text { Spectral } \\
\text { Esficiency }\end{array}$} & \multirow[t]{2}{*}{$\nu$} & \multicolumn{4}{|c|}{ Gains (BER) } \\
\hline & & & & $10 \%$ & $5 \%$ & $2 \%$ & $1 \%$ \\
\hline BPSK & uneoded & 1 & - & $1.3(2.6)$ & $0.9(2.5)$ & $0.6(2.3)$ & $0.5(2.2)$ \\
\hline BPSK & $1 / 2$ & $1 / 2$ & 2 & $1.3(1.6)$ & $0.9(1.2)$ & $0.7(0.9)$ & $0.5(0.8)$ \\
\hline BPSK & $1 / 2$ & $1 / 2$ & 3 & $1.1(1.5)$ & $0.9(1.1)$ & $0.7(0.9)$ & $0.5(0.8)$ \\
\hline BPSK & $1 / 2$ & $1 / 2$ & 4 & $1.2(1.7)$ & $1.0(1.4)$ & $0.8(1.1)$ & $0.5(1.0)$ \\
\hline BPSK & $2 / 3$ & $2 / 3$ & 2 & $2.0(3.0)$ & $1.5(2.4)$ & $1.1(2.1)$ & $.9(1.8)$ \\
\hline BI'SK & $2 / 3$ & $2 / 3$ & 3 & $1.6(2.1)$ & $1.1(2.1)$ & $1.1(1.8)$ & $.9(1.5)$ \\
\hline BPSK & $2 / 3$ & $2 / 3$ & 4 & $2.1(3.2)$ & $1.8(2.7)$ & $1.3(2.3)$ & $1.1(1.9)$ \\
\hline QLSK & $1 / 2$ & 1 & 2 & $1.4(1.6)$ & $0.9(1.2)$ & $0.7(1.0)$ & $0.6(0.9)$ \\
\hline QPSK & $1 / 2$ & 1 & 3 & $1.1(1.5)$ & $0.8(1.1)$ & $0.7(0.9)$ & $0.5(0.8)$ \\
\hline $\mathrm{QL}^{2} \mathrm{SK}$ & $1 / 2$ & 1 & 4 & $1.3(1.6)$ & $1.0(1.3)$ & $0.8(1.1)$ & $0.6(1.0)$ \\
\hline 8PSK & $2 / 3$ & 2 & 3 & $1.9(2.3)$ & $1.5(2.1)$ & $1.1(1.8)$ & $1.0(1.7)$ \\
\hline 8PSK & $2 / 3$ & 2 & 1 & $2.8(3.5)$ & $2.2(2.8)$ & $1.8(2.4)$ & $1.5(2.1)$ \\
\hline
\end{tabular}

TABLE IV

MAP Decoding Gains for Different Trellis Codes over AWGN (Rayleigh) Channels. Source III [with Distribution $\operatorname{Pr}(0 \mid 0)=.9, \operatorname{Pr}(1 \mid 1)=.8]$

\begin{tabular}{c|c|c|c||c|c|c|c}
\hline $\begin{array}{c}\text { Modulation } \\
\text { Type }\end{array}$ & Rate & $\begin{array}{c}\text { Spectral } \\
\text { Efficiency }\end{array}$ & \multicolumn{1}{|c||}{$\nu$} & \multicolumn{4}{|c}{ Gains (BEI $)$} \\
\hline \hline BPSK & uncoded & 1 & - & $2.3(4.2)$ & $1.5(3.8)$ & $1.0(3.6)$ & $0.8(3.5)$ \\
\hline \hline BPSK & $1 / 2$ & $1 / 2$ & 2 & $1.9(2.6)$ & $1.4(1.9)$ & $1.0(1.5)$ & $0.9(1.3)$ \\
\hline BPSK & $1 / 2$ & $1 / 2$ & 3 & $1.9(2.5)$ & $1.3(1.9)$ & $1.0(1.4)$ & $0.8(1.3)$ \\
\hline BPSK & $1 / 2$ & $1 / 2$ & 4 & $2.1(2.8)$ & $1.5(2.2)$ & $1.2(1.8)$ & $1.0(1.5)$ \\
\hline BPSK & $2 / 3$ & $2 / 3$ & 2 & $3.5(4.9)$ & $2.6(4.1)$ & $2.0(3.4)$ & $1.6(3.0)$ \\
\hline BPSK & $2 / 3$ & $2 / 3$ & 3 & $2.9(4.2)$ & $2.2(3.4)$ & $1.8(2.8)$ & $1.6(2.4)$ \\
\hline BPSK & $2 / 3$ & $2 / 3$ & 4 & $3.5(4.9)$ & $2.8(4.1)$ & $2.1(3.5)$ & $1.8(3.0)$ \\
\hline \hline QPSSK & $1 / 2$ & 1 & 2 & $1.9(2.5)$ & $1.4(1.9)$ & $1.0(1.6)$ & $0.8(1.4)$ \\
\hline QPSK & $1 / 2$ & 1 & 3 & $1.8(2.4)$ & $1.3(1.8)$ & $1.0(1.4)$ & $0.8(1.2)$ \\
\hline QPSK & $1 / 2$ & 1 & 4 & $2.1(2.7)$ & $1.5(2.1)$ & $1.2(1.6)$ & $0.9(1.5)$ \\
\hline 8PSK & $2 / 3$ & 2 & 3 & $3.3(4.1)$ & $2.6(3.5)$ & $2.0(3.1)$ & $1.7(2.9)$ \\
\hline 8PSK & $2 / 3$ & 2 & 4 & $4.6(5.6)$ & $3.5(4.5)$ & $2.7(3.8)$ & $2.3(3.4)$ \\
\hline
\end{tabular}

TABLE V

MAP Decoding Gains for Different Trellis Codes over AWGN (Rayleigh) Channels. Source IV [with Distribution $\operatorname{Pr}(0 \mid 0)=.2, \operatorname{Pr}(1 \mid 1)=.9$ ]

\begin{tabular}{c|c|c|c|c|c|c|c}
\hline $\begin{array}{c}\text { Modulation } \\
\text { Type }\end{array}$ & Rate & $\begin{array}{c}\text { Spectral } \\
\text { Efficiency }\end{array}$ & $\nu$ & \multicolumn{4}{|c}{ Gains (BER) } \\
\hline \hline BPSK & uncoded & 1 & - & $2.2(5.0)$ & $1.1(3.2)$ & $0.7(2.7)$ & $0.6(2.5)$ \\
\hline \hline BPSK & $1 / 2$ & $1 / 2$ & 2 & $2.6(3.6)$ & $1.6(2.3)$ & $1.1(1.6)$ & $0.9(1.3)$ \\
\hline BPSK & $1 / 2$ & $1 / 2$ & 3 & $2.5(3.5)$ & $1.6(2.4)$ & $1.2(1.7)$ & $1.0(1.5)$ \\
\hline BPSK & $1 / 2$ & $1 / 2$ & 1 & $2.9(3.9)$ & $1.9(2.7)$ & $1.4(2.0)$ & $1.2(1.7)$ \\
\hline \hline BPSK & $2 / 3$ & $2 / 3$ & 2 & $6.7(8.9)$ & $3.4(5.0)$ & $2.2(3.6)$ & $1.7(3.0)$ \\
\hline BISK & $2 / 3$ & $2 / 3$ & 3 & $6.5(8.7)$ & $3.4(5.0)$ & $2.3(3.6)$ & $1.8(2.9)$ \\
\hline BPSK & $2 / 3$ & $2 / 3$ & 4 & $6.3(8.5)$ & $3.7(5.4)$ & $2.5(4.0)$ & $2.0(3.3)$ \\
\hline \hline QLSK & $1 / 2$ & 1 & 2 & $2.9(3.7)$ & $1.6(2.2)$ & $1.1(1.5)$ & $0.9(1.3)$ \\
\hline QPSK & $1 / 2$ & 1 & 3 & $2.5(3.5)$ & $1.6(2.2)$ & $1.1(1.6)$ & $0.9(1.3)$ \\
\hline QPSK & $1 / 2$ & 1 & 4 & $2.9(3.9)$ & $1.9(2.6)$ & $1.4(2.0)$ & $1.1(1.7)$ \\
\hline 8PSK & $2 / 3$ & 2 & 3 & $7.5(9.4)$ & $4.4(5.4)$ & $3.0(4.0)$ & $2.4(3.5)$ \\
\hline 8PSK & $2 / 3$ & 2 & 4 & $7.7(9.4)$ & $4.7(5.9)$ & $3.3(4.6)$ & $2.7(3.8)$ \\
\hline
\end{tabular}

Signal Costellation

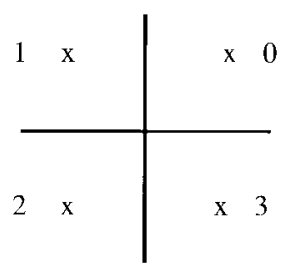

4-D Signal Points

$\mathrm{S}(0=(0,0) \quad \mathrm{S} 1=(0,2)$

$\mathrm{S} 2=(1,1) \quad \mathrm{S} 3=(1,3)$

$\mathrm{S} 4=(2,2) \quad \mathrm{S} 5=(2,0)$

$\mathrm{S} 6=(3,3) \quad \mathrm{S} 7=(3,1)$

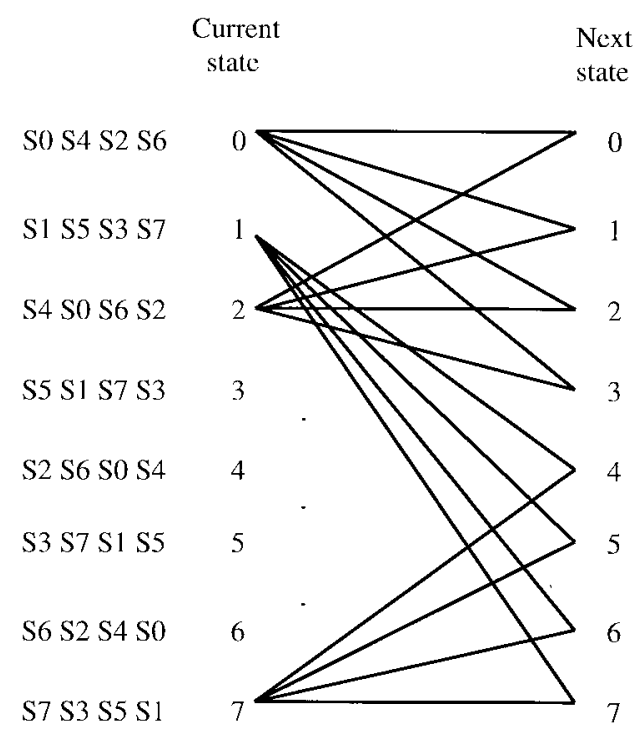

Fig. 3. Four-dimensional signal points and the trellis diagram of the 4-D QPSK eight-state encoder.

also be observed that the gains monotonically increase with increasing the total redundancy; i.e., the effect of redundancy type is not apparent as in uncoded systems. This is attributed to the fact that convolutional codes are constructed using finite state machines which introduce memory to the system. Even for sources with no memory, the coded bits will have some memory correlation. 
Regarding the number of encoder memory elements, it can be seen that the major portion of decoding gains can be obtained using four-state codes. Although more complex codes improve the performance at low error rates, they do not significantly improve the performance at relatively high error rates. This is because codes with more states suffer more from error propagation at very high channel error rates.

The effect of increasing the encoder rate is clear from the simulation results for rate $2 / 3$ codes with BPSK modulation-higher decoding gains are obtained. For example, for Source IV a decoding gain of 2.2 (resp. 3.6) dB at a BER of 0.02 is obtained over the AWGN (resp. Rayleigh) channel with a four-state rate $2 / 3$ code, while the four-state rate $1 / 2$ code achieves a decoding gain of only 1.1 (resp. 1.6) $\mathrm{dB}$ at the same BER and source model over the AWGN (resp. Rayleigh) channel. Increasing the encoder rate further will also yield higher decoding gains. Another (perhaps surprising) observation is that the performance of the eight-state code is slightly worse than the performance of the four-state code at high error rates. This is because three memory elements are used and one of the bits has only one delay element in the encoder structure. As a result, the four-state code will outperform the eight-state code at high error rates. This fact has an effect on the decoding gains for the two codes, and in some instances the decoding gains of the four-state code are slightly higher than the gains for the eight-state code.

Finally, the effect of increasing the signal constellation is shown in the results for the 8-PSK-coded systems. For example, at a rate of $2 / 3$, the decoding gains for the 8 -PSK systems are higher than the gains for the same rate with BPSK modulation. This is because the signal constellation has more points and hence it is more sensitive to large noise values. This fact of increased MAP decoding gains is desirable since as the demand increases for systems with higher spectral efficiencies, and hence higher signal constellations, more MAP decoding gains could be obtained. Decoding gains as much as $3.8 \mathrm{~dB}$ at a BER of only 0.01 are achieved (see Table V).

\section{E. The Effect of Increasing the Signal Dimensionality on MAP Gains}

The previous results suggest that as the encoder rate is increased, the benefit of MAP decoding (relative to ML) increases. In trellis codes with multidimensional constellations (i.e., dimension greater than two-also referred to as multiple TCM), the information bits are grouped and sent over $m$ signaling intervals. For example, in the previously mentioned 2-D Gray-mapped QPSK (2-D QPSK) one information bit is delivered to a rate $1 / 2$ encoder every signal interval. The two encoder output bits are mapped to one of the QPSK signals. The bandwidth efficiency is $1 \mathrm{~b} / \mathrm{s} / \mathrm{Hz}$. Suppose we have a 4-D QPSK scheme with the same bandwidth efficiency and ML coding gains. What is the performance of the two codes with sequence MAP decoding? The previous results suggest that the 4-D QPSK-coded system will perform better.

A comparative example is used here to show the effect of increasing the signal dimensionality on the sequence MAP decoding gains. The previously used eight-state Gray-mapped QPSK code has a minimum time diversity of four. Its normalized minimum Euclidean and product distances are 12 and 64, respectively. If we compare this code with the codes in [21] and [22], we realize that it is optimized for both AWGN and Rayleigh channels. An eight-state 4-D code is constructed. Its trellis diagram is shown in Fig. 3. Two information bits are delivered to a rate $2 / 3$ encoder every two signal intervals. The encoder's 3-b output is used to select one of the eight twotuple QPSK signal points. The bandwidth efficiency of this code is also $1 \mathrm{~b} / \mathrm{s} / \mathrm{Hz}$, and its minimum time diversity is four. Its normalized minimum Euclidean and product distances are 12 and 64, respectively-i.e., its asymptotic coding gain with ML decoding is the same as that of the 2-D QPSK scheme. Fig. 4 shows the performance of the two codes with ML decoding and source model IV over the Rayleigh channel. If sequence MAP decoding is used, the performance of the two codes is different, especially at low SNR. Fig. 5 show the performance of the two codes with MAP decoding. It is clear that the 4-D QPSK scheme outperforms the corresponding 2-D QPSK scheme. Increasing the signal dimensionality is also expected to yield more gains. However, since this will also increase the number of signals per trellis branch, more delay and interleaving are required which may not be suitable for real-time applications.

\section{CELP LSP's CODING}

Codebook-excited linear predictive coding is a frameoriented technique that breaks a speech signal into blocks of samples that are processed as one unit. The particular implementation we consider is Federal Standard 1016 (FS 1016) 4.8-kb/s CELP [23]. The CELP parameters that are transmitted over the noisy channel include the stochastic codebook index and gain, the adaptive codebook index (pitch delay) and gain, and ten ordered LSP's. In the FS 1016 CELP, each LSP is quantized by either a 3-b or a 4-b scalar quantizer. The second through fifth LSP's are quantized by 4-b quantizers; the rest are quantized to $3 \mathrm{~b}$. The quantized LSP's refer to frequencies that must be ordered (LSP-1 < LSP-2 $<\cdots<$ LSP-10). In this work, we consider only the three most significant bits of each LSP, ignoring the least significant bit in the second through fifth parameters.

The CELP encoder leaves some redundancy in the encoded bit stream in the form of memory and nonuniformity. The modeling of CELP-encoded speech is described in detail in [6]. This modeling is briefly described below. A large training sequence consisting of 83826 frames (about $42 \mathrm{~min}$ of speech) from the TIMIT speech database [11] was used to estimate the prior LSP distributions needed for the MAP decoding. For every $30 \mathrm{~ms}$ of speech, an LPC analysis was performed according to FS 1016 standards to arrive at the ten quantized LSP's. The relative frequency of transitions between the values of the three high-order bits of each LSP were compiled to extract Markov transition probabilities.

Suppose we encode a segment of speech using FS 1016 CELP, resulting in a sequence of CELP frames. The quantized LSP frames are approximated by a (block) stationary Markov 


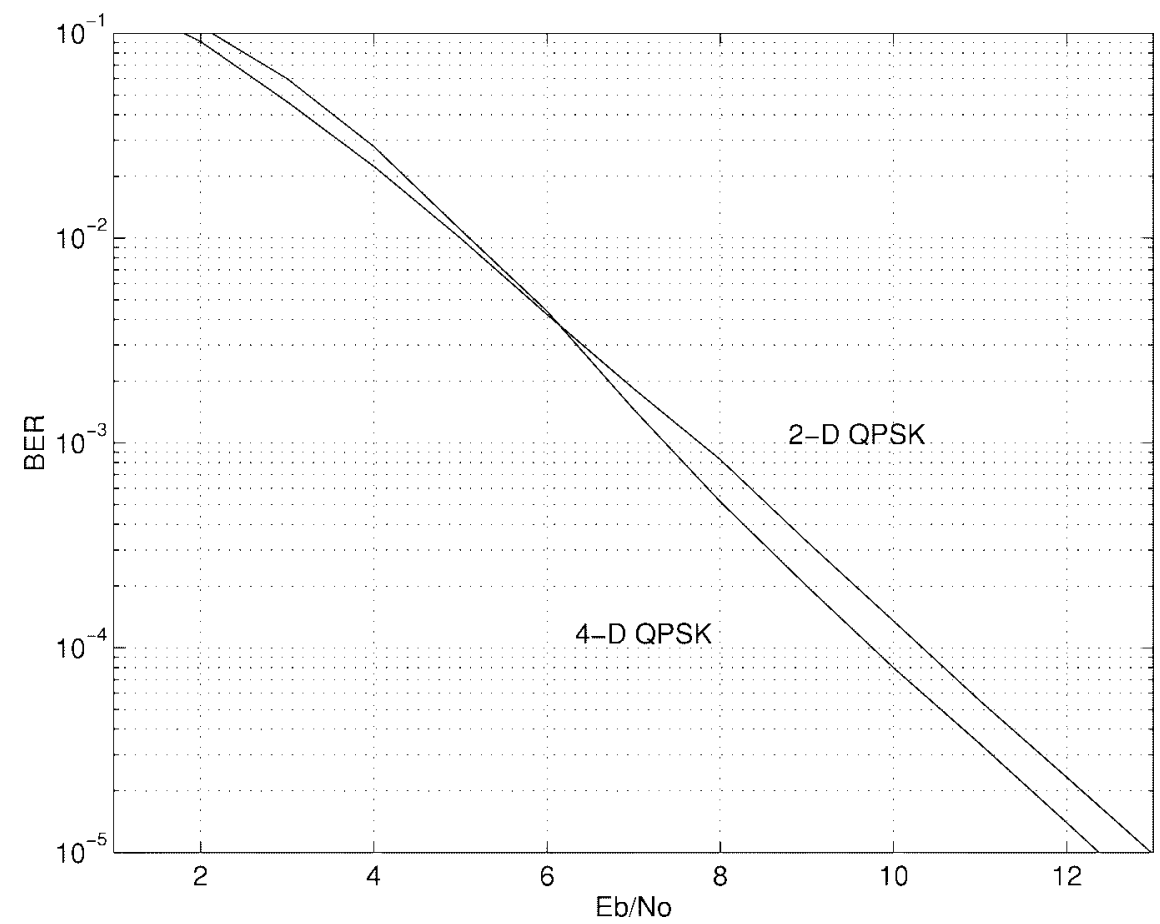

Fig. 4. BER of the 2-D and 4-D QPSK eight-state schemes with ML decoding.

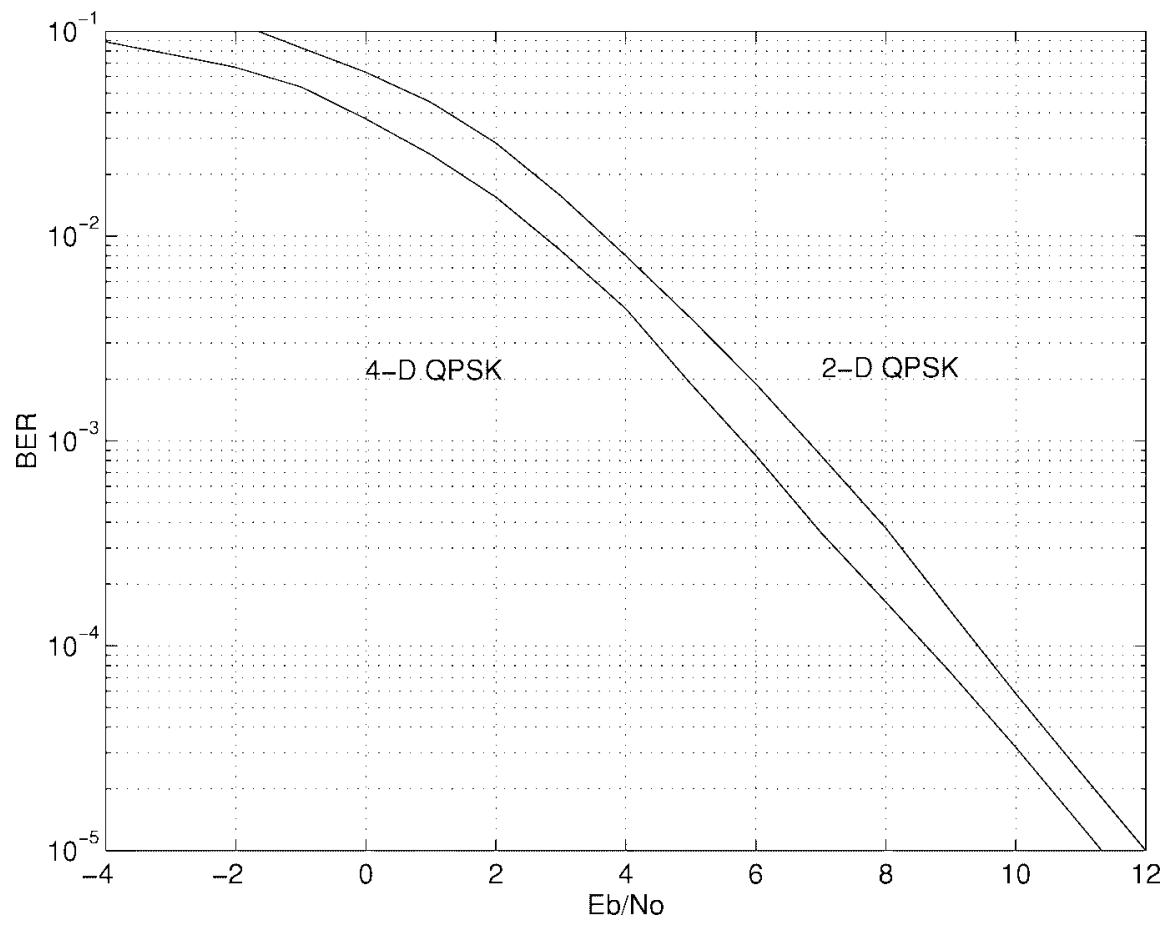

Fig. 5. BER of the 2-D and 4-D QPSK eight-state schemes with MAP decoding.

process [6]. Denote the process entropy rate (in bits/frame) by $H_{\infty}$ which represents the minimum number of bits per frame required to describe three bits. The CELP encoder produces $30 \mathrm{~b} /$ frame to describe the LSP's, so the residual redundancy-i.e., the total redundancy (per frame) in the CELP-encoded LSP's-is

$$
\rho_{T}=30-H_{\infty} \text { (bits/frame) }
$$

$H_{\infty}$ (and so $\rho_{T}$ ) were estimated by observing a long training sequence and matching the observations to a particular model of a random process; the entropy rate of the model process was then computed and used as our estimate of $H_{\infty}$.

Two models for the generation of LSP's distribution are proposed.

- Model 1-which does not attempt to take into account any correlation between frames-indicates that $\rho_{T}=9.867$ 
of the 30 high-order bits in the LSP's are redundant. Approximately $\rho_{D}=5.275$ bits of redundancy were due to the nonuniform distribution of the LSP's, and approximately $\rho_{M}=4.593$ bits of redundancy were due to the memory within a frame.

- Model 2-which does take into account both interframe and intraframe correlation-indicates that $\rho_{T}=12.485$ of the 30 high-order bits in the LSP's are redundant. Once again, $\rho_{D}=5.275$ bits of redundancy were due to the nonuniform distribution of the LSP's, while $\rho_{M}=7.211$ bits of redundancy were due to the memory remaining both within a frame and between frames.

We employ three soft-decision decoding schemes based on the Viterbi decoding algorithm.

- ML-a maximum likelihood Viterbi decoding algorithm which does not utilize the LSP's a priori information.

- MAP 1-a maximum a posteriori (MAP) decoding algorithm that exploits only the redundancy due to the nonuniform distribution of the LSP's and their correlation within a frame-approximately $10 \mathrm{~b} /$ frame.

- MAP 2-which exploits the redundancy from the nonuniform distribution of the LSP's and their interframe and intraframe correlation-approximately $12.5 \mathrm{~b} /$ frame.

A decoding buffer length of ten symbols is used to limit the decoding delay. All algorithms are implemented so as to yield a decoding delay of only one frame.

\section{A. Coding of the LSP's}

Since the quantized LSP's are represented by 3 b, every binary three-tuple representing one LSP must be encoded every encoder time unit. In [6], a rate 3/4 binary convolutional code with BPSK modulation was used. The resulting system spectral efficiency was $0.75 \mathrm{~b} / \mathrm{s} / \mathrm{Hz}$. To increase the spectral efficiency we propose using QPSK modulation. The proposed codes are four-state and eight-state rate 3/4 codes with 4-D QPSK. Their spectral efficiency is $1.5 \mathrm{~b} / \mathrm{s} / \mathrm{Hz}$. These codes were designed for Rayleigh fading channels [24]. Moreover, their minimum Euclidean distances are the same as the corresponding codes designed for the AWGN channel [21]. This means that both codes are optimum for both channels. The minimum Euclidean distances of the four-state and eight-state codes are six and eight, respectively. The minimum time diversity of both codes are two. However, the minimum product distance of the eightstate code is 16 , which is twice that of the four-state code. It should be pointed out that the number of branches in the minimum-length error event path for the four-state code is two while that of the eight-state code is one (i.e., corresponds to parallel branches). The effect of this will be clear from the simulations.

\section{B. Simulation Results}

Simulations were used to determine the performance of the proposed decoding algorithms. The three high-order bits of each of the ten quantized LSP's were channel encoded using the two codes described above. The outputs of the channel encoders were then mapped to a pair of QPSK signals and transmitted over either the AWGN channel or the fully
TABLE VI

Sequence MAP Decoding Gains For the CELP-Encoded SPEech WITH Four-STATE 4-D QPSK TCM SCHEMES OVER Both AWGN and RaYleigh ChanNels

\begin{tabular}{|c|c|c|c|c|c|c|c|c|c|}
\hline \multirow{2}{*}{$\begin{array}{c}\text { Decoding } \\
\text { Gains }\end{array}$} & \multirow{2}{*}{$\begin{array}{c}\text { Channel } \\
\text { Type }\end{array}$} & \multicolumn{4}{|c|}{$S D(d B)$} & \multicolumn{4}{|c|}{$P_{s}(\%)$} \\
\hline & & 2.0 & 2.5 & 3.0 & 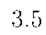 & 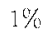 & $5 \%$ & $10 \%$ & \\
\hline & 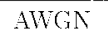 & & 1.5 & & & & 1 & & .6 \\
\hline & & & 2. & & & $\pi$ & .1 & & \\
\hline & & & & & & & & & \\
\hline s. M. & Ra: & & 0 & & & & 6 & & \\
\hline & & 1.5 & 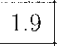 & 2.2 & & .0 & 14 & & \\
\hline vs. ML. & Rayle & 3.1 & 3.5 & 3.7 & & 2.3 & 2.6 & 2.8 & \\
\hline
\end{tabular}

TABLE VII

Sequence MAP Decoding Gains for the CELP-Encoded SPEECH WITH EIGHT-STATE 4-D QPSK TCM SCHEMES OVER Both AWGN and RAYLEIGH ChanNels

\begin{tabular}{|c|c|c|c|c|c|c|c|c|c|}
\hline \multirow{2}{*}{$\begin{array}{l}\text { Decoding } \\
\text { Cains }\end{array}$} & \multirow{2}{*}{$\begin{array}{c}\text { Channel } \\
\text { Type }\end{array}$} & \multicolumn{4}{|c|}{$\mathrm{SD}(\mathrm{dB})$} & \multicolumn{4}{|c|}{$P_{s}(\%)$} \\
\hline & & 2.0 & 2.5 & 3.0 & 3.5 & $1 \%$ & $5 \%$ & $10 \%$ & $15 \%$ \\
\hline $\mathrm{MAP} 1$ & AWGA & 1.1 & 1.3 & \begin{tabular}{|l}
1.6 \\
\end{tabular} & 1.8 & 0.7 & 1.0 & 1.2 & 1.3 \\
\hline vs. ML & Rayleigh & 2.3 & 2.4 & 2.6 & 2.9 & 1.9 & 1.9 & 2.0 & 2.1 \\
\hline MAP 2 & AWGN & 0.4 & 0.5 & 0.5 & 0.6 & 0.2 & 0.3 & 0.4 & 0.4 \\
\hline vs. MAP' 1 & Rayleigh & 0.8 & \begin{tabular}{|l|}
0.8 \\
\end{tabular} & 0.8 & 0.9 & 0.5 & 0.6 & 0.6 & 0.6 \\
\hline MAP 2 & AWGN & 1.5 & 1.8 & 2.1 & 2.4 & 0.9 & 1.3 & 1.6 & 1.7 \\
\hline vs. $\mathrm{Ml}_{i}$ & Rayleigh & 3.1 & 3.2 & 3.4 & 3.8 & 2.4 & 2.5 & 2.6 & 2.7 \\
\hline
\end{tabular}

interleaved Rayleigh channel. After appropriate demodulation, the signals were decoded with the proposed channel decoders and the decoded LSP's were fed into the CELP decoder for speech reconstruction. The decoder buffer length is set to ten symbols [24]. This allows interleaving to be done within only one frame, and so only a delay of one frame exists. Note that sequence MAP decoding does not introduce any additional delay.

The testing sequence consisted of 4753 frames (about 2.2 min of speech) - 48 sentences, half uttered by female speakers and half by male speakers from different dialect regions. No speaker appeared in both the training and testing sequences. Thus, the approach used in this simulation was to use a single "universal" model-constructed from a very large training sequence-to decode all the speech samples.

In evaluating the performance of the various decoders, we use two criteria. The first is the average spectral distortion $(S D)$, the most commonly used distortion measure for the LSP's [25]. More specifically

$$
\begin{aligned}
S D=\frac{1}{T} \sum_{j=1}^{T}[ & \int_{-\pi}^{\pi}\left(10 \log _{10} S_{j}(w)\right. \\
& \left.\left.\quad-10 \log _{10} \hat{S}_{j}(w)\right)^{2} \frac{d w}{2 \pi}\right]^{\frac{1}{2}} \mathrm{~dB}
\end{aligned}
$$

where $S_{j}(w)$ and $\hat{S}_{j}(w)$ are the original and reconstructed spectra associated with frame $j$ and $T$ is the total number of frames. Roughly speaking, an average spectral distortion of 1 $\mathrm{dB}$ or less is equivalent to perceptually transparent encoding of the LSP coefficients [25]. In addition to average spectral distortion, the percentage of outliers-i.e., the fraction of 


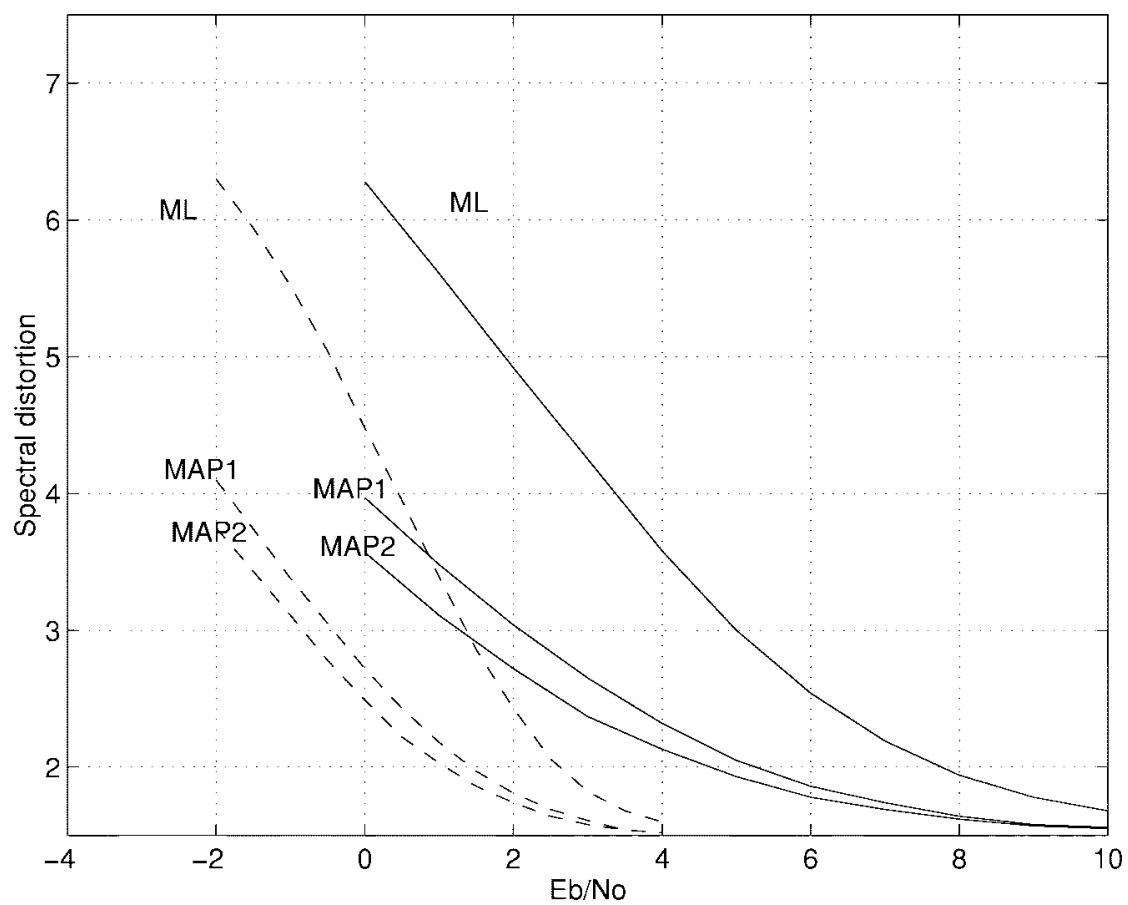

Fig. 6. Spectral distortion versus $E_{b} / N_{o}$ for the four-state 4-D QPSK scheme: solid (Rayleigh) and dashed (AWGN).

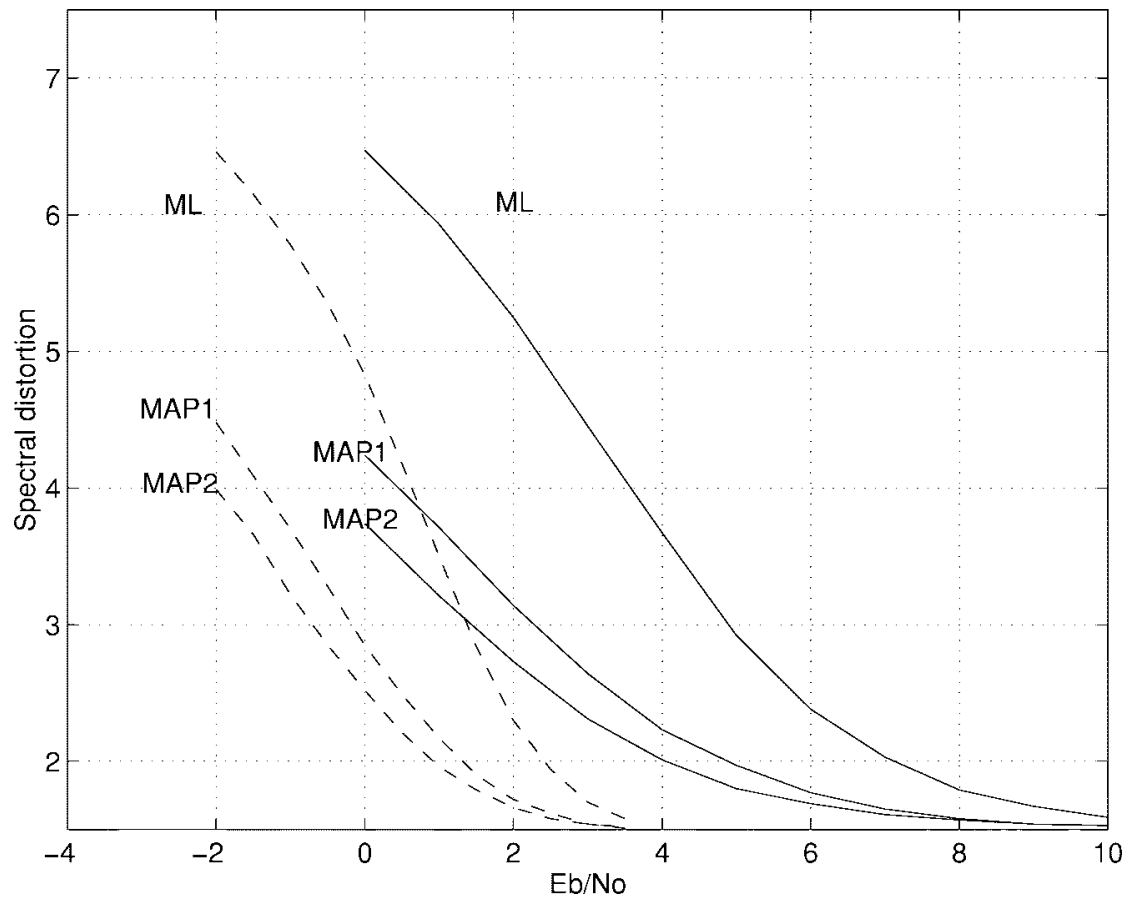

Fig. 7. Spectral distortion versus $E_{b} / N_{o}$ for the eight-state 4-D QPSK scheme: solid (Rayleigh) and dashed (AWGN).

frames with distortion greater than $4 \mathrm{~dB}$-was also compiled during the simulation. It should be noted that the spectral distortion introduced by CELP's scalar quantizer alone (when the channel is noiseless) is around $1.50 \mathrm{~dB}$ with $0.08 \%$ of outliers $>4 \mathrm{~dB}$.

The second measure of the decoders' performance is symbol error rate $\left(P_{s}\right)$, i.e., the fraction of LSP's the decoder decoded incorrectly. Tables VI and VII indicate the MAP decoding gains for the two codes over both channels. The gains are shown for a $P_{s}$ values of $1 \%, 5 \%, 10 \%$, and $15 \%$, since error concealment and interpolation are made at $P_{s}$ 's as high as $15 \%$.

It is clear that the gains over the Rayleigh channel are higher than the gains over the AWGN channel. Moreover, significant gains are achieved. For example, at an average spectral distortion of $2 \mathrm{~dB}$, total MAP decoding gains of 1.5 and $3.1 \mathrm{~dB}$ were achieved over the AWGN and Rayleigh channels, respectively. Spectral distortion gains are shown 


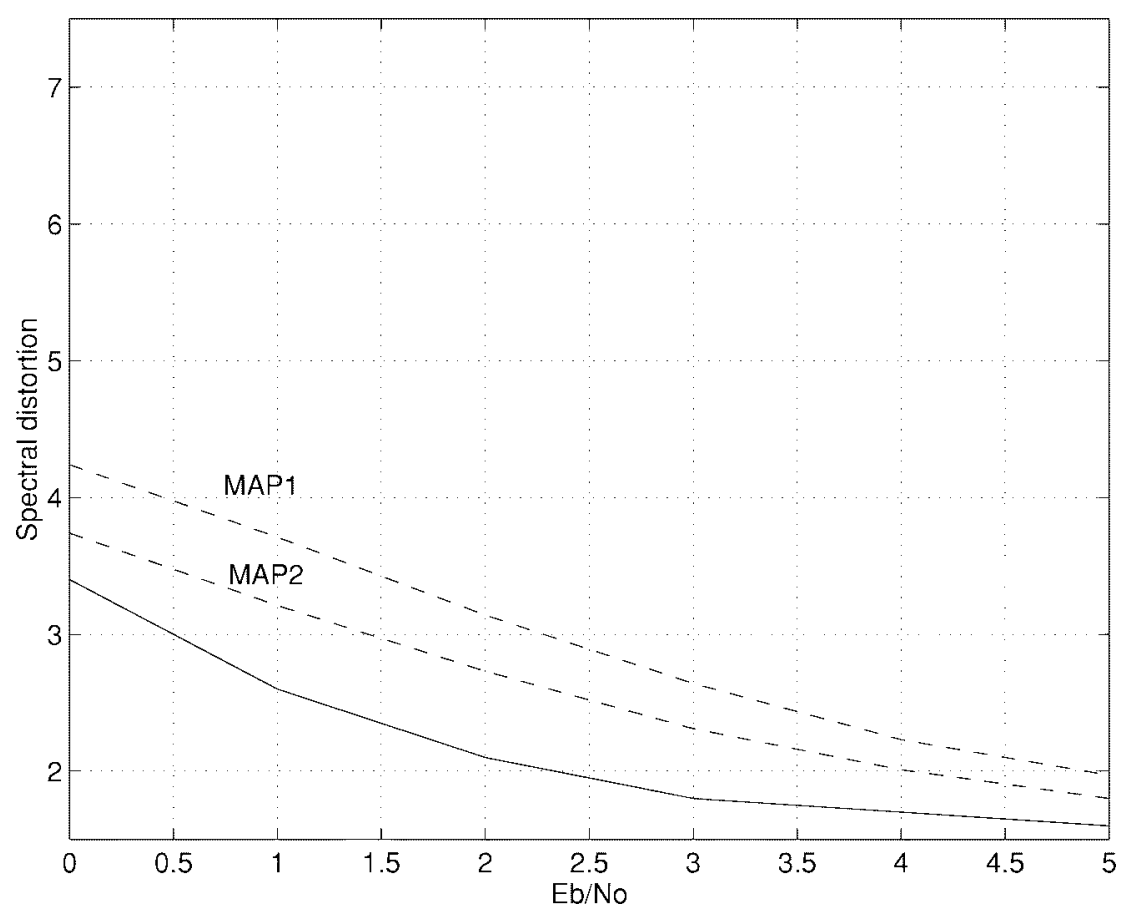

Fig. 8. Spectral distortion versus $E_{b} / N_{o}$ for the eight-state 4-D QPSK scheme (dashed) and the I-Q QPSK 32-state code (solid) over the Rayleigh channel.

TABLE VIII

SEQUence MAP 1 DeCoding Gains FOR THE CELP-ENCODED SPEECH WITH 32-STATE I-Q QPSK TCM SCHEME over Both AWGN and RaYleigh ChanNels

\begin{tabular}{c|c|c|c|c|c|c|c|c|c}
\hline Decoding & Channel & \multicolumn{4}{|c|}{ SD(dB) } & \multicolumn{4}{c}{$P_{s}(\%)$} \\
\cline { 3 - 11 } Gains & Type & 2.0 & 2.5 & 3.0 & 3.5 & $1 \%$ & $5 \%$ & $10 \%$ & $15 \%$ \\
\hline MAT 1 & AWGN & 2.1 & 2.4 & 2.5 & 1.8 & 0.7 & 1.0 & 1.2 & 1.3 \\
\cline { 2 - 10 } vs. ML & Rayleigh & 3.7 & 4.1 & 4.2 & 4.5 & 3.7 & 4.1 & 1.4 & 1.6 \\
\hline \hline
\end{tabular}

in Figs. 6 and 7. Also, Tables VI and VII summarize the decoding gains for various $\mathrm{SD}$ and $P_{s}$ values. It is noticed that the large portion of the gains can be achieved using Model 1 . This agrees with the calculation of the redundancies for both models, where the second model gives only 2.5 -b additional redundancy. A comparison between the two codes shows that even though the eight-state code outperforms the four-state code at high SNR's, the MAP decoding gains for the fourstate code are slightly higher. The reason for that is attributed to the codes' minimum error event path length, which affects the performance at low SNR.

\section{Coding of CELP LSP's via I-Q QPSK}

In this section, another coding scheme is proposed. In [26], it was shown that so-called "I-Q" trellis codes-in which the in-phase and quadrature components of the transmitted signal are encoded independently - can offer higher minimum time diversities. Also, the increased encoder rate gives higher decoding gains. These two features are used together to provide a robust system with a bandwidth efficiency of $1.5 \mathrm{~b} / \mathrm{s} / \mathrm{Hz}$. The proposed scheme is outlined as follows. Two frames are encoded together using two rate $3 / 4$ encoders. Each encoder is mapped to a binary antipodal signal. The first frame is encoded using encoder I and the second frame is encoded using encoder Q. The transmitted signal is QPSK where its in phase (quadrature) are specified by encoder I (encoder Q). Only Model 1 for the a priori LSP's information is used (i.e., correlation between frames is not exploited) and a delay of two frames is imposed. The same 32 -state rate $3 / 4$ code proposed in [6] is used. Using this approach, larger MAP decoding gains are obtained. Table VIII displays the decoding gains obtained using this scheme. Also, a comparison between this code and the previous ones in Fig. 8 shows that the I-Q code is achieves a very robust performance at very noisy channel conditions.

\section{CONCLUSION}

In this paper, we considered sequence maximum a posteriori (MAP) decoding of correlated signals transmitted over very noisy AWGN and Rayleigh channels. A first-order twostate Markov model is used for the source. A variety of different systems with different sources, BPSK, QPSK, and 8-PSK modulation schemes and different encoder complexities were simulated. Sequence MAP decoding (compared to ML decoding) proves to substantially improve the performance at very noisy channel conditions, especially for systems with moderate redundancy. The MAP decoding gains when the channel is Rayleigh distributed are higher than the gains when the channel is AWGN. Most of the MAP decoding (relative to ML decoding) gains are achieved with low complexity encoders. Moreover, trellis-coded systems with higher encoder rates have significantly more MAP decoding gains. Also, more decoding gains are obtained for encoders with larger signal constellations. A comparative example of a 4-D versus a 2-D constellations shows that the multidimensional constellation achieves more MAP gains than its corresponding trellis-coded scheme with 2-D constellation. 
Trellis encoding the CELP LSP's with 4-D QPSK modulation is also presented. Two source models are used. One is based on the intraframe correlation while the second one models both intraframe and interframe correlations. Coding gains as much as $4 \mathrm{~dB}$ are achieved. Finally, a comparison between the conventionally designed codes and an I-Q QPSK scheme shows that the I-Q scheme achieves better performance although only the correlation within a frame is exploited.

\section{REFERENCES}

[1] C. E. Shannon, "A mathematical theory of communication" Bell Syst. Tech. J., vol. 27, pp. 379-423, July 1948.

[2] N. Farvardin and V. Vaishampayan, "Optimal quantizer design for noisy channels: An approach to combined source-channel coding," IEEE Trans. Inform. Theory, vol. 33, pp. 827-838, Nov. 1987.

[3] E. Ayanoglu and R. Gray, "The design of joint source and channel trellis waveform coders," IEEE Trans. Inform. Theory, vol. 33, pp. 885-865, Nov. 1987.

[4] N. Farvardin, "A study of vector quantization for noisy channels," IEEE Trans. Inform. Theory, vol. 36, pp. 799-809, July 1990.

[5] J. Hagenauer, "Source controlled channel decoding," IEEE Trans. Commun., vol. 43, pp. 2449-2457, Sept. 1995.

[6] F. Alajaji, N. Phamdo, and T. Fuja, "Channel codes that exploit the residual redundancy in CELP-encoded speech," IEEE Trans. Speech Audio Processing, vol. 4, pp. 325-336, Sept. 1996.

[7] J. Kroll and N. Phamdo, "Analysis and design of trellis codes optimized for a binary symmetric Markov source with maximum a posteriori detection," IEEE Trans. Inform. Theory, vol. 44, pp. 2977-2987, Nov. 1998.

[8] C. Hartmann and L. Rudolph, "An optimum symbol-by-symbol decoding rule for linear codes," IEEE Trans. Inform. Theory, vol. $22 \mathrm{pp}$. 514-517, Sept. 1976.

[9] L. Bahl, J. Cocke, F. Jelinek, and J. Raviv, "Optimal decoding of linear codes for minimizing symbol error rate," IEEE Trans. Inform. Theory, vol. 20, pp. 284-287, Mar. 1974.

[10] P. McAdam, L. Welch, and C. Weber, "M.A.P. bit decoding of convolutional codes," in IEEE Int. Symp. Information Theory, Asilomar, CA, Jan. 1972

[11] National Institute of Standards and Technology (NIST), "The DARPA TIMIT acoustic-phonetic continuous speech corpus CD-ROM,” Oct. 1990.

[12] F. Alajaji, N. Phamdo, N. Farvardin, and T. Fuja, "Detection of binary Markov sources over channels with additive Markov noise," IEEE Trans. Inform. Theory, vol. 42, pp. 230-239, Jan. 1996.

[13] E. Biglieri, D. Divsalar, P. Mclane, and M. Simon, Introduction to Trellis-Coded Modulation with Applications. New York: Macmillan, 1991.

[14] A. Viterbi and J. Omura, Principles of Digital Communications and Coding. New York: McGraw-Hill, 1979.

[15] M. Best, M. Burnashev, Y. Levy, A. Rabinovich, P. Fishburn, A. Calderbank, and D. Costello, "On a techniques to calculate the exact performance of a convolutional codes," IEEE Trans. Inform. Theory, vol. 41, pp. 441-447, Mar. 1995.

[16] S. Pietrobon, "On the probability of error of convolutional codes," IEEE Trans. Inform. Theory, vol. 42, pp. 1562-1568, Sept. 1996.

[17] M. Burnashev, "Improved union bound for Viterbi decoder of convolutional codes," in Proc. 1995, Int. Symp. Information Theory, Whistler, Canada, Sept. 1995, p. 167.

[18] S. Lin and D. J. Costello, Jr., Error Control Coding: Fundamentals and Applications. Englewood Cliffs, NJ: Prentice-Hall, 1983.

[19] G. Ungerboeck, "Channel coding with multilevel/phase signals," IEEE Trans. Inform. Theory, vol. 28, pp. 55-67, Jan. 1982.

[20] C. Schlegel and D. J. Costello, Jr., "Bandwidth efficient coding for fading channels: Code construction and performance analysis," IEEE J. Select. Areas Commun., vol. 7, pp. 1356-1368, Dec. 1989.

[21] S. Pietrobon, R. Deng, A. Lafanchere, G. Ungerboeck, and D. Costello Jr., "Trellis-coded multidimensional phase modulation," IEEE Trans. Inform. Theory, vol. 36, pp. 63-88, Jan. 1990.

[22] E. Leonardo, L. Zheng, and B. Vucetic, "Multidimensional MPSK trellis codes for fading channels," IEEE Trans. Inform. Theory, vol. 42, pp. 1093-1108, July 1996.

[23] National Communications System (NCS), "Details to assist in implementation of Federal Standard 1016 CELP," Tech. Inform. Bull. 92-1, Arlington, VA.
[24] L.-F. Wei, "Coded M-DPSK with built-in time diversity for fading channels," IEEE Trans. Inform. Theory, vol. 39, pp. 1820-1839, Nov. 1993.

[25] R. Laroia, N. Phamdo, and N. Farvardin, "Robust and efficient quantization of speech LSP parameters using structured vector quantization," in Proc. ICASSP-91, pp. 661-664.

[26] S. Al-Semari and T. Fuja, "I-Q TCM: Reliable communication over the Rayleigh fading channel close to the cutoff rate," IEEE Trans. Inform. Theory, vol. 43, pp. 250-262, Jan. 1997.

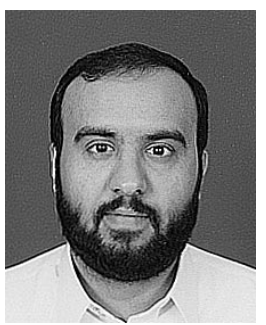

Saud A. Al-Semari was born on December 25, 1968. He received the B.S. and M.Sc. degrees in electrical engineering from King Fahd University of Petroleum \& Minerals (KFUPM), Dhahran, Saud Arabia, in 1991 and 1992, respectively. He received the Ph.D. degree from the University of Maryland, College Park, in 1995.

Since 1996, he has been an Assistant Professor of Electrical Engineering at KFUPM. He is also a Division Manager at the KFUPM Research Institute. His current research interests lie in coded modulation, error control coding, and wireless communications systems and networks.

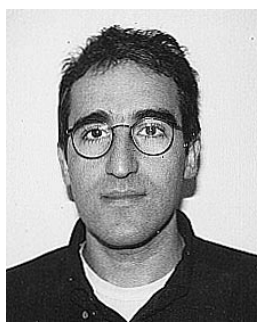

Fady Alajaji (S'90-M'94) was born in Beirut, Lebanon, on May 1, 1966. He received the B.E. degree (with Distinction) from the American University of Beirut, Lebanon, and the M.S. and Ph.D. degrees from the University of Maryland, College Park, all in electrical engineering, in 1988, 1990, and 1994, respectively.

He held a graduate Fellowship from 1990 to 1992 and a post-doctoral appointment in 1994 at the Institute for Systems Research, University of Maryland. In 1995, he joined the Department of Mathematics and Statistics, Queen's University, Kingston, Ont., Canada, where he is an Assistant Professor of Mathematics and Engineering. Since 1997, he has also been crossappointed as an Assistant Professor in the Department of Electrical and Computer Engineering, Queen's University. His research interests include information theory, digital communications, error control coding, joint source-channel coding, and data compression.

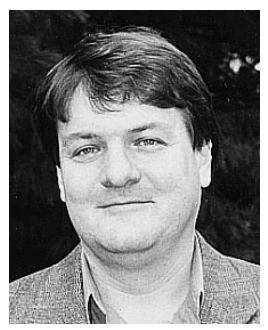

Tom Fuja (S'80-M'91-SM'97) received the B.S.E.E. and B.S.Comp.E. degrees in 1981 from the University of Michigan, Ann Arbor, and the M.Eng and $\mathrm{Ph} . \mathrm{D}$. degrees in electrical engineering in 1983 and 1987, respectively, from Cornell University, Ithaca, NY.

In 1982 and 1983, he was a Member of the Technical Staff at AT\&T Bell Laboratories, Holmdel, NJ. In 1987, he joined the faculty of the University of Maryland, College Park, where he held a joint appointment as an Associate Professor in the Electrical Engineering Department and the Institute for Systems Research. In 1997 and 1998, he served in a visiting position as Program Director for communications research at the National Science Foundation. In August 1998, he joined the University of Notre Dame, Notre Dame, IN, as an Associate Professor of Electrical Engineering. His research interests lie in the area of digital communications systems - specifically coding theory and applications and information theory. Most of his recent research has focused on coding for wireless applications and on the interface between source coding and channel coding.

Dr. Fuja was the recipient of a Presidential Young Investigator award from the National Science Foundation in 1989. In 1991, he received the George Corcoran Memorial Award for his significant contribution to electrical engineering education at the University of Maryland. He has been active in the IEEE Information Theory Society and is currently a member of its Board of Governors. 